\title{
Antikaon nuclear potential in hot and dense matter
}

\author{
Laura Tolós, Angels Ramos, and Artur Polls \\ Departament d'Estructura i Constituents de la Matèria, Universitat de Barcelona, Diagonal 647, E-08028 Barcelona, Spain
}

(Received 19 February 2002; published 29 April 2002)

\begin{abstract}
The antikaon optical potential in hot and dense nuclear matter is studied within the framework of a coupledchannel self-consistent calculation taking, as bare meson-baryon interaction, the meson-exchange potential of the Jülich group. Typical conditions found in heavy-ion collisions at GSI are explored. As in the case of zero temperature, the angular momentum components larger than $L=0$ contribute significantly to the finite temperature antikaon optical potential at finite momentum. It is found that the particular treatment of the medium effects has a strong influence on the behavior of the antikaon potential with temperature. Our self-consistent model, in which antikaons and pions are dressed in the medium, gives a moderately temperature dependent antikaon potential which remains attractive at GSI temperatures, contrary to what one finds if only nuclear Pauli blocking effects are included.
\end{abstract}

DOI: 10.1103/PhysRevC.65.054907

PACS number(s): 13.75.Jz, 25.75.-q, 21.30.Fe, 21.65.+f

\section{INTRODUCTION}

The study of the properties of hadrons in hot and dense matter has received a lot of attention in the last years, in connection to the experimental programs at SIS-GSI, SPSCERN, RHIC-BNL, and the forthcoming operation of LHC [1]. A particular effort has been invested in understanding the properties of antikaons due to the direct implications they have in astrophysical phenomena. If the antikaon mass drops in matter below the electron chemical potential, a condensed fraction of $K^{-}$would appear [2], giving rise to a softer nuclear equation of state and to a substantial reduction of the maximum mass that neutron stars could sustain (see updated references in Ref. [3]).

There have been some attempts to extract the antikaonnucleus potential from a best-fit analysis of kaonic-atom data, and some solutions favor very strongly attractive well depths of the order of $200 \mathrm{MeV}$ at normal nuclear matter density [4]. However, recent self-consistent calculations based on a chiral lagrangian [5-7] or meson-exchange potentials [8] only predicted moderate attractive depths of 50-80 MeV. In addition, studies of kaonic atoms using the chiral $\bar{K} N$ amplitudes of Ref. [9] showed that it is indeed possible to find a reasonable reproduction of the data with a relatively shallow antikaon-nucleus potential [10], albeit adding an additional moderate phenomenological piece [11]. This was recently corroborated by a calculation [12], where a good fit to both scattering $K^{-} p$ data and kaonic-atom data only required a slight modification at the parameters of the chiral meson-baryon interaction model of Ref. [13]. The lesson learned is that kaonic atom data do not really provide a suitable constraint on the antikaon-nucleus potential at normal nuclear matter density.

Heavy-ion collisions can also offer information on the modification of the properties of hadrons. In particular, the enhanced $K^{-} / K^{+}$ratio measured at GSI [14] can be understood assuming that the antikaons feel a strong attraction [15-18], although other interpretations in terms of the enhanced cross sections due to the shifting of the $\Lambda(1405)$ resonance to higher energies have also been advocated [7], a mechanism that was already suggested in Ref. [19] to explain the data of $K^{-}$absorption at rest on ${ }^{12} \mathrm{C}[20,21]$.

In fact, the antikaon-nucleon amplitude in isospin $I=0$ is dominated by the presence of this $\Lambda(1405)$ resonant structure, which in free space appears only $27 \mathrm{MeV}$ below the $\bar{K} N$ threshold. The resonance is generated dynamically from a $T$-matrix scattering equation in coupled channels using a suitable meson-baryon potential. The coupling between the $\bar{K} N$ and $\pi Y(Y=\Lambda, \Sigma)$ channels is essential to obtain the right dynamical behavior in free space. Relatedly, the inmedium properties of the $\Lambda(1405)$ such as its position and its width, which in turn influence strongly the behavior of the antikaon-nucleus optical potential, are very sensitive to the many-body treatment of the medium effects. Previous works have shown, for instance, that a self-consistent treatment of the $\bar{K}$ self-energy had a strong influence on the scattering amplitudes [5-7] and, consequently, on the in-medium properties of the antikaon. The incorporation of the pion with its medium modified properties also proved to be important [6], although most works until now have ignored it $[5,7,8,12]$.

In our previous work [8] we performed a study of the antikaon properties in the medium within a self-consistent dynamical model which used, as bare interaction, the mesonexchange potential of the Jülich group [22]. Since antikaons are produced at finite momentum, we focused on the effect of high partial waves, typically excluded in the chiral models $[5,6]$. We note, however, that $p$ waves were recently incorporated into the chiral amplitudes $[23,24]$, and a first estimation of their contribution to the in-medium properties of the antikaons was also recently reported [25].

One must keep in mind that heavy-ion collisions produce dense and hot matter. Therefore, to learn about the meson properties in this environment, it is necessary to incorporate in the formalism not only the medium effects but also those associated with having a finite nonzero temperature. The purpose of the present work is, precisely, to develop a proper self-consistent calculation of the antikaon properties in a dense and hot medium, extending to finite $T$ our previous $T=0$ model [8]. We will indeed show that the antikaon properties at finite $T$ depend very strongly on the treatment of the 
medium. The present work focuses on typical scenarios found in heavy-ion collisions at GSI. For this reason, results will be shown up to densities of twice the nuclear saturation density and up to temperatures of $70 \mathrm{MeV}$.

We have organized the present paper as follows. In Sec. II we first review our formalism at $T=0$ and include some updated ingredients, such as the incorporation of the pion self-energy and the use of some slightly different prescriptions for the single-particle energies of the baryons. Next we show how we treat the temperature effects, which amounts to using the appropriate finite $T$ nucleon Pauli blocking factor, as well as using temperature dependent self-energies for the mesons (antikaons and pions) and the nucleons. Some lengthy derivations are relegated to the Appendix. Our results are presented and discussed in Sec. III, and concluding remarks are given in Sec. IV.

\section{FORMALISM}

In this section we review the calculation of the singleparticle potential of the $\bar{K}$ meson at $T=0$ in symmetric nuclear matter. In addition to the self-consistent incorporation of the $\bar{K}$ self-energy, as done in our previous work [8], here we also include the self-energy of the pion in the intermediate $\pi \Lambda, \pi \Sigma$ states present in the construction of the $\bar{K} N$ effective interaction, since this was shown to be important in Ref. [6]. In a further step, we extend the model to finite temperature, which affects the Pauli blocking of the nucleons in the intermediate states, as well as the dressing of mesons and baryons.

\section{A. Revision of the $T=0$ formalism}

In Ref. [8], the effective $\bar{K} N$ interaction in the nuclear medium ( $G$ matrix) at $T=0$ was derived from a mesonbaryon bare interaction built in the meson exchange framework [22]. As the bare interaction permits transitions from the $\bar{K} N$ channel to the $\pi \Sigma$ and $\pi \Lambda$ ones, all having a strangeness $S=-1$, we are confronted with a coupledchannel problem. Working in isospin coupled basis, the $\bar{K} N$ channel can have isospin $I=0$ or $I=1$, so the resultant $G$ matrices are classified according to the value of isospin. For $I=0, \bar{K} N$ and $\pi \Sigma$ are the only channels available, while for $I=1$ the $\pi \Lambda$ channel is also allowed. In a schematic notation, each $G$-matrix fulfills the coupled-channel equation

$$
\begin{aligned}
\left\langle M_{1} B_{1}|G(\Omega)| M_{2} B_{2}\right\rangle= & \left\langle M_{1} B_{1}|V(\sqrt{s})| M_{2} B_{2}\right\rangle \\
& +\sum_{M_{3} B_{3}}\left\langle M_{1} B_{1}|V(\sqrt{s})| M_{3} B_{3}\right\rangle \\
& \times \frac{Q_{M_{3} B_{3}}}{\Omega-E_{M_{3}}-E_{B_{3}}+i \eta} \\
& \times\left\langle M_{3} B_{3}|G(\Omega)| M_{2} B_{2}\right\rangle,
\end{aligned}
$$

where $\Omega$ is the so-called starting energy, given in the lab frame, and $\sqrt{s}$ is the invariant center-of-mass energy. In Eq.
(1), $M_{i}$ and $B_{i}$ represent, respectively, the possible mesons $(\bar{K}, \pi)$ and baryons $(N, \Lambda, \Sigma)$, and their corresponding quantum numbers, such as coupled spin and isospin, and linear momentum. The function $Q_{M_{3} B_{3}}$ stands for the Pauli operator preventing the nucleons in the intermediate states from occupying already filled ones.

The prescription for the single-particle energies of all the mesons and baryons participating in the reaction and in the intermediate states is written as

$$
E_{M_{i}\left(B_{i}\right)}(k)=\sqrt{k^{2}+m_{M_{i}\left(B_{i}\right)}^{2}}+U_{M_{i}\left(B_{i}\right)}\left(k, E_{M_{i}\left(B_{i}\right)}^{q p}\right),
$$

where $U_{M_{i}\left(B_{i}\right)}$ is the single-particle potential of each meson (baryon) calculated at the real quasiparticle energy $E_{M_{i}\left(B_{i}\right)}^{q p}$. For baryons, this quasiparticle energy is given by

$$
E_{B_{i}}^{q p}(k)=\sqrt{k^{2}+m_{B_{i}}^{2}}+U_{B_{i}}(k),
$$

while, for mesons, it is obtained by solving the equation

$$
\left(E_{M_{i}}^{q p}(k)\right)^{2}=k^{2}+m_{M_{i}}^{2}+\operatorname{Re} \Pi_{M_{i}}\left(k, E_{M_{i}}^{q p}\right),
$$

where $\Pi_{M_{i}}$ is the meson self-energy.

The $\bar{K}$ single-particle potential in the Brueckner-HartreeFock approach is schematically given by

$$
U_{\bar{K}}\left(k_{\bar{K}}, E_{\bar{K}}^{q p}\right)=\sum_{N \leqslant F}\left\langle\bar{K} N\left|G\left(\Omega=E_{N}^{q p}+E_{\bar{K}}^{q p}\right)\right| \bar{K} N\right\rangle,
$$

where the summation over nucleon states is limited by the nucleon Fermi momentum. The $\bar{K}$ self-energy is obtained from the optical potential through the relation

$$
\Pi_{\bar{K}}\left(k_{\bar{K}}, \omega\right)=2 \sqrt{k_{\bar{K}}^{2}+m_{\bar{K}}^{2}} U_{\bar{k}}\left(k_{\bar{K}}, \omega\right) .
$$

As can be easily seen from Eq. (5), since the $\bar{K} N$ effective interaction (the $G$ matrix) depends on the $\bar{K}$ single-particle energy, which in turn depends on the $\bar{K}$ potential through Eq. (6), we are confronted with a self-consistency problem.

We proceed as in Ref. [8], where self-consistency for the optical potential was demanded at the quasiparticle energy. After self-consistency is reached, the complete $\bar{K}$ energyand momentum-dependent self-energy of the $\bar{K}$ can be obtained, from which the $\bar{K}$ propagator

$$
D_{\bar{K}}\left(k_{\bar{K}}, \omega\right)=\frac{1}{\omega^{2}-k_{\bar{K}}^{2}-m_{\bar{K}}^{2}-\Pi_{\bar{K}}\left(k_{\bar{K}}, \omega\right)}
$$

and the corresponding spectral density, defined as

$$
S_{\bar{K}}\left(k_{\bar{K}}, \omega\right)=-\frac{1}{\pi} \operatorname{Im} D_{\bar{K}}\left(k_{\bar{K}}, \omega\right),
$$

can be derived. We note that our self-consistent procedure amounts to replacing, in the $\bar{K}$ propagator, the energy- 
dependent self-energy, $\Pi_{\bar{K}}\left(k_{\bar{K}}, \omega\right)$ with that evaluated at the quasiparticle energy, $\Pi_{\bar{K}}\left[k_{\bar{K}}, \omega=E_{\bar{K}}^{q p}\left(k_{\bar{K}}\right)\right]$. This is what we refer to as the quasiparticle self-consistent approach, which retains the position and width of the peak of the $\bar{K}$ spectral function at each iteration. This simplification versus the more complete self-consistent job done in Refs. [5,6] allows one to perform analytically the energy integral of the intermediate loops, thus reducing the four-dimensional integral equation to a three-dimensional one.

In this paper, our model at $T=0$ has been reviewed by implementing some modifications in the properties of the baryons and mesons. First, we have introduced slight modifications in the single-particle potential of the $\Lambda$ and $\Sigma$ hyperons, following the parametrization of Ref. [26],

$$
U_{\Lambda, \Sigma}(\rho)=-340 \rho+1087.5 \rho^{2} \text {, }
$$

which is more appropriate in the high-density regime than the simple parametrization, linear in $\rho$, used previously.

For nucleons, we have used a relativistic $\sigma-\omega$ model, where the scalar and vector coupling constants, $g_{s}$ and $g_{v}$ respectively, are density dependent [27]. This is a simple way to mimic results from Dirac-Brueckner-Hartree-Fock calculations.

The most important modification comes from the introduction of the pion self-energy $\Pi_{\pi}\left(k_{\pi}, \omega\right)$ in the intermediate $\pi \Sigma, \pi \Lambda$ states present in the construction of the $\bar{K} N$ effective interaction. The pion is dressed with the momentum and energy-dependent self-energy already used in Ref. [6]. This self-energy incorporates a $p$-wave piece built up from the coupling to $1 p-1 h, 1 \Delta-1 h$ and $2 p-2 h$ excitations, plus short-range correlations. The model also contains a small and constant $s$-wave part (see the Appendix for details and appropriate references of the model).

As in the case of the $\bar{K}$ meson, we can define a pion optical potential, $U_{\pi}\left(k_{\pi}, \omega\right)$, from the complete $\pi$ selfenergy. We use

$$
U_{\pi}\left(k_{\pi}, \omega\right)=\sqrt{k_{\pi}^{2}+m_{\pi}^{2}+\Pi_{\pi}\left(k_{\pi}, \omega\right)}-\sqrt{k_{\pi}^{2}+m_{\pi}^{2}},
$$

which ensures that, when inserted in the $G$-matrix equation, one is using a quasiparticle approximation to the spectral function, with the peak located at the right quasiparticle energy defined in Eq. (4). Due to the small mass of the pion, it has not been possible to retain only the first term in the expansion of the first square root of Eq. (10), as we did for the $\bar{K}$ meson.

The spectral density for $\pi$ is calculated from Eqs. (7) and (8), by replacing the $\bar{K}$ properties for those of the $\pi$.

\section{B. Temperature effects}

The introduction of temperature in the calculation of the $G$-matrix affects the Pauli blocking of the intermediate nucleon states, as well as the dressing of mesons and baryons. The $G$-matrix equation at finite $T$ reads formally as in Eq. (1), but replacing

$$
\begin{gathered}
Q_{M B} \rightarrow Q_{M B}(T), \\
G(\Omega) \rightarrow G(\Omega, T), \\
E_{M}, \quad E_{B} \rightarrow E_{M}(T), \quad E_{B}(T) .
\end{gathered}
$$

The function $Q_{M B}(T)$ is unity for meson-hyperon states while, for $\bar{K} N$ states, it follows the law $1-n\left(k_{N}, T\right)$, where $n\left(k_{N}, T\right)$ is the nucleon Fermi distribution at the corresponding temperature

$$
n\left(k_{N}, T\right)=\frac{1}{1+\exp \left(\left[E_{N}\left(k_{N}, T\right)-\mu\right] / T\right)} .
$$

The nucleonic spectrum at finite $T, E_{N}\left(k_{N}, T\right)$, is obtained following a $\sigma-\omega$ model that will be described below and $\mu$ is the chemical potential obtained imposing the normalization property

$$
\rho=\frac{\nu}{(2 \pi)^{3}} \int d^{3} k_{N} n\left(k_{N}, T\right),
$$

at each density $\rho$, where $\nu=4$ is the degeneration factor of symmetric nuclear matter.

As in the $T=0$ case, we perform an angle average of the Pauli operator $Q_{\bar{K} N}(T)$, a strategy which facilitates the solution of the $G$-matrix equation in a partial wave basis. We first define $\vec{P}$ and $\vec{k}$ as the total and relative momenta of the $\bar{K} N$ pair, respectively,

$$
\vec{P}=\vec{k}_{\bar{K}}+\vec{k}_{N}, \quad \vec{k}=\frac{m_{N} \vec{k}_{\bar{K}}-m_{\bar{K}} \vec{k}_{N}}{m_{\bar{K}}+m_{N}},
$$

which allows us to rewrite the nucleon and antikaon momenta in the laboratory system, $\vec{k}_{N}$ and $\vec{k}_{\bar{K}}$, as

$$
\vec{k}_{N}=-\vec{k}+\frac{\xi}{1+\xi} \vec{P}, \quad \vec{k}_{\bar{K}}=\vec{k}+\frac{1}{1+\xi} \vec{P},
$$

where $m_{\bar{K}}$ and $m_{N}$ are the kaon and nucleon masses, respectively, and $\xi=m_{N} / m_{\bar{K}}$. In terms of the total and relative momenta, the Pauli operator $Q_{\bar{K} N}\left(k_{N}, T\right)$ reads $Q_{\bar{K} N}(\mid[\xi /(1$ $+\xi)] \vec{P}-\vec{k} \mid, T)$, which explicitly shows the dependence on the angle between $\vec{P}$ and $\vec{k}$. This dependence is eliminated in the $G$-matrix equation by replacing $Q_{\bar{K} N}$ by its angle average

$$
\bar{Q}_{\bar{K} N}(P, k, T)=\frac{1}{2} \int_{-1}^{1} d x Q_{\bar{K} N}(\vec{P}, \vec{k}, T)=\frac{1}{2 B} \ln \frac{e^{A}+e^{B}}{e^{A}+e^{-B}},
$$

where 


$$
\begin{gathered}
A=\frac{\mu}{T}-\frac{k^{2}+\left(\frac{\xi}{\xi+1}\right)^{2} P^{2}}{2 m_{N} T}, \\
B=\frac{k \xi P}{T(1+\xi) m_{N}} .
\end{gathered}
$$

Temperature also affects the properties of the particles involved in the process. The $\bar{K}$ optical potential at a given temperature is calculated according to

$$
\begin{aligned}
U_{\bar{K}}\left(k_{\bar{K}}, E_{\bar{K}}^{q p}, T\right)= & \int d^{3} k_{N} n\left(k_{N}, T\right) \\
& \times\left\langle\bar{K} N\left|G\left(\Omega=E_{N}^{q p}+E_{\bar{K}}^{q p}, T\right)\right| \bar{K} N\right\rangle .
\end{aligned}
$$

Once more, this is a self-consistent problem for $U_{\bar{K}}$. More explicitly, using the partial-wave components of the $G$ matrix, we obtain (similarly to Ref. [8])

$$
\begin{aligned}
U_{\bar{K}}\left(k_{\bar{K}}, E_{\bar{K}}^{q p}, T\right)= & \frac{1}{2} \sum_{L, J, I}(2 J+1)(2 I+1) \int n\left(k_{N}, T\right) k_{N}^{2} d k_{N} \\
& \times\left\langle\bar{k}\left|G^{L J I}\left[\bar{P}, E_{\bar{K}}^{q p}\left(k_{\bar{K}}\right)+E_{N}^{q p}\left(k_{N}\right), T\right]\right| \bar{k}\right\rangle,
\end{aligned}
$$

where $\bar{k}$ and $\bar{P}$ are the relative and center-of-mass momenta, respectively, averaged over the angle between the external $\bar{K}$ momentum in the lab system, $k_{\bar{K}}$, and the internal momentum of the nucleon, $k_{N}$. In the actual calculations, we include partial waves up to $L=4$.

We also have to pay attention to the temperature effects on the properties of the other hadrons participating in the process. We have to be especially careful with the pion, since its small mass makes it very sensitive to variations in its properties. As mentioned before, the pion self-energy at $T$ $=0$ has been obtained following a model that includes the coupling to $1 p-1 h, 1 \Delta-1 h$, and $2 p-2 h$ excitations. The details on how this model is modified by the effect of a finite temperature are presented in the Appendix.

In the case of nucleons, we have introduced temperature effects following the Walecka $\sigma-\omega$ model [28], using the density dependent scalar and vector coupling constants at $T$ $=0$ given in Ref. [27]. We have obtained the baryonic chemical potential $\mu$, the effective mass $m^{*}$, and the vector potential $\Sigma^{0}$ simultaneously, fixing the temperature and the density. The value of $\mu$ is defined via the baryonic density $\rho$ [see Eqs. (11) and (12)], where $E_{N}\left(k_{N}, T\right)$ is the nucleonic energy spectrum,

$$
E_{N}\left(k_{N}, T\right)=\sqrt{k_{N}^{2}+m^{*}(T)^{2}}-\Sigma^{0},
$$

and $\Sigma_{0}$ and $m^{*}$ are defined as

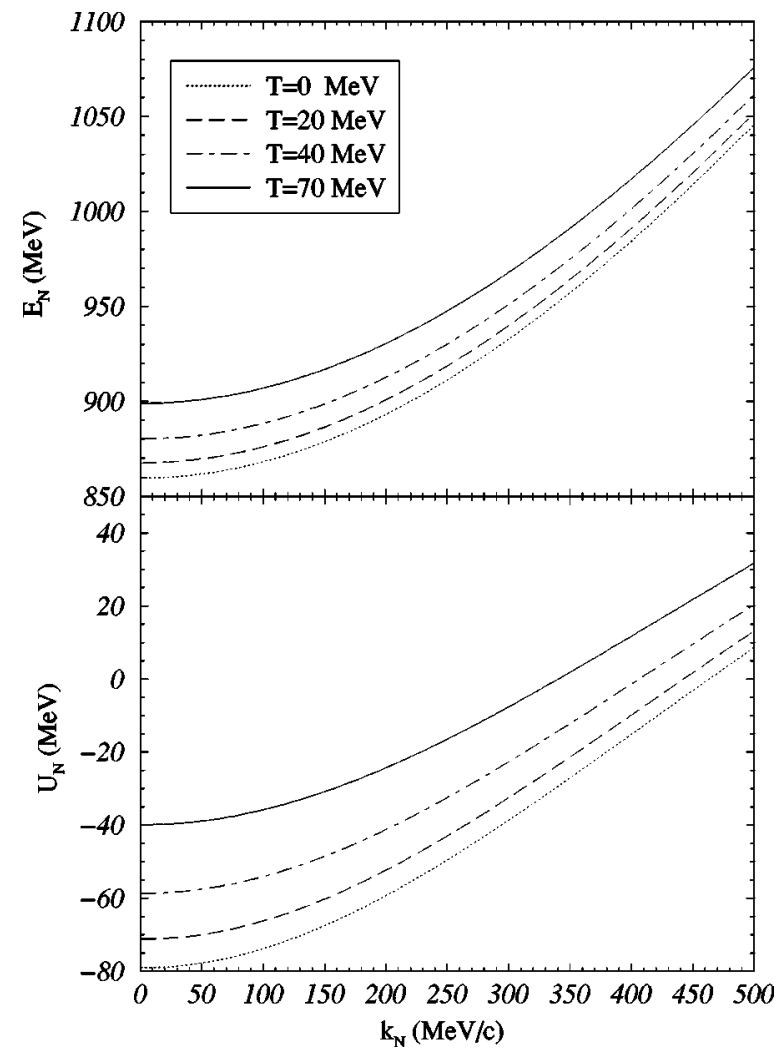

FIG. 1. Nucleon energy spectrum and nucleon potential at $\rho$ $=0.17 \mathrm{fm}^{-3}$ as a function of the nucleon momentum for different temperatures.

$$
\begin{gathered}
\Sigma^{0}=-\left(\frac{g_{v}}{m_{v}}\right)^{2} \rho, \\
m^{*}(T)=m-\left(\frac{g_{s}}{m_{s}}\right)^{2} \frac{\nu}{(2 \pi)^{3}} \int d^{3} k_{N} \frac{m^{*}(T)}{\sqrt{k_{N}^{2}+m^{*}(T)^{2}}} n\left(k_{N}, T\right) .
\end{gathered}
$$

One clearly sees that, given $g_{s}$ and $g_{v}$ at $T=0$, and a fixed $\rho$, $\Sigma^{0}$ is easily obtained. On the other hand, a simultaneous solution of $\mu$ and $m^{*}$ is needed to determine the nucleonic spectrum.

We note that the antiparticle contribution to the density $\rho$ has been proven to be negligible, so it is not considered here. We have not considered either changes in the hyperon properties induced by the use of a finite temperature. We have checked, in a schematic Skyrme-Hartree-Fock calculation, that these changes are small within the temperature range explored in this work.

\section{RESULTS}

We start this section by studying the effect of the temperature on the nucleon spectrum and the pion self-energy, both of them crucial ingredients for the calculation of $U_{\bar{K}}$. In Fig. 1 , the nucleon spectrum and the nucleon potential, defined as $U_{N}=E_{N}-\sqrt{k_{N}^{2}+m_{N}^{2}}$, are given as functions of the nucleon momentum for various temperatures at the nuclear saturation 


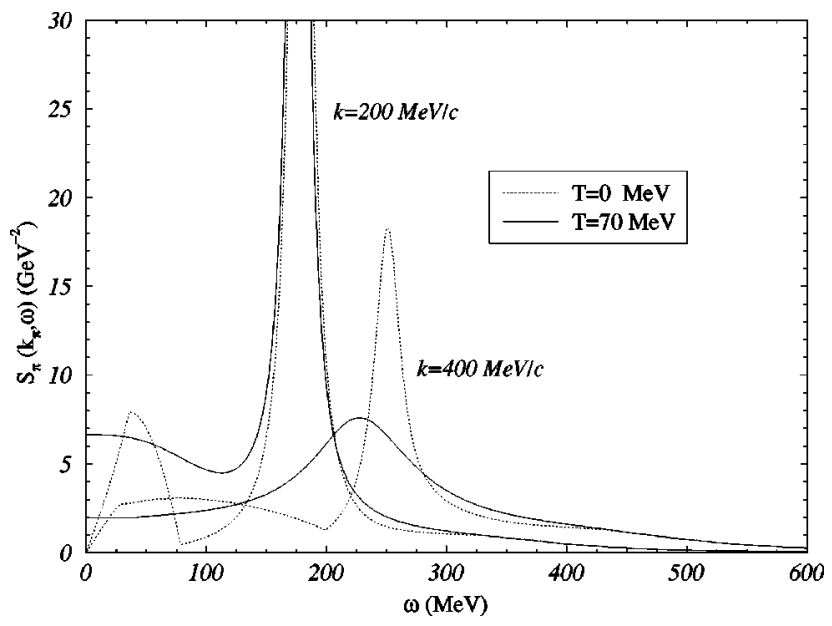

FIG. 2. Spectral density of the pion at $\rho=0.17 \mathrm{fm}^{-3}$ as a function of energy for $k_{\pi}=200 \mathrm{MeV} / c$ and $k_{\pi}=400 \mathrm{MeV} / c$, and for $T=0$ and $70 \mathrm{MeV}$.

density $\rho_{0}=0.17 \mathrm{fm}^{-3}$. The lowest curves in both graphs correspond to $T=0$ and 4 , as the value of the temperature increases up to $T=70 \mathrm{MeV}$ (solid lines), the attractive potential is reduced from around $-80 \mathrm{MeV}$ to $-40 \mathrm{MeV}$ at $k_{N}=0$. Consequently, the energy spectrum for $k_{N}=0$ goes from 860 to $900 \mathrm{MeV}$. A similar trend is observed for higher values of the nucleon momentum. This behavior is well known for the $\sigma-\omega$ models, as reported in Ref. [28].

The next two figures give us information about the pion self-energy and how it is modified by temperature. Figure 2 displays the spectral density of the pion as a function of energy at nuclear saturation density, $\rho_{0}=0.17 \mathrm{fm}^{-3}$, for two pion momenta $k_{\pi}=200$ and $400 \mathrm{MeV}$, and two different temperatures $T=0 \mathrm{MeV}$ (dotted lines) and $T=70 \mathrm{MeV}$ (solid lines). At $T=0$, the structure of the $1 p-1 h$ excitations can be seen very clearly on the left side of the quasiparticle peak. This structure smooths out as temperature increases. The effect of $1 \Delta-1 h$ excitations is more difficult to identify for these two momenta. It is only somewhat appreciated at $T$ $=0$ by a slower fall of the spectral function with energy to the right of the quasiparticle peak. The effect of temperature is to move the quasiparticle peak slightly away from the $T$ $=0$ position toward lower energies, making it noticeably wider.

In Fig. 3 we show the pion optical potential as defined in Eq. (10). The real and imaginary parts of the pion optical potential at nuclear saturation density are displayed as functions of the pion momentum $k_{\pi}$ for different temperatures. The dotted lines correspond to $T=0$, and the results for the highest temperature studied, $T=70 \mathrm{MeV}$, are represented by the solid ones. In the region of pion momenta explored here, the imaginary part shows a stronger dependence on the temperature than the real part, which is practically $T$ independent. Note that the quasiparticle energy, which defines the position of the quasiparticle peak in the spectral function, is determined through the real part of the pion self-energy [see Eq. (4)], and it is not directly obtained from $\operatorname{Re} U_{\pi}$, where $U_{\pi}$ is given in Eq. (10). This explains that, while $\operatorname{Re} U_{\pi}$ at $k_{\pi}=400 \mathrm{MeV}$ is practically the same for $T=0$ and $70 \mathrm{MeV}$,

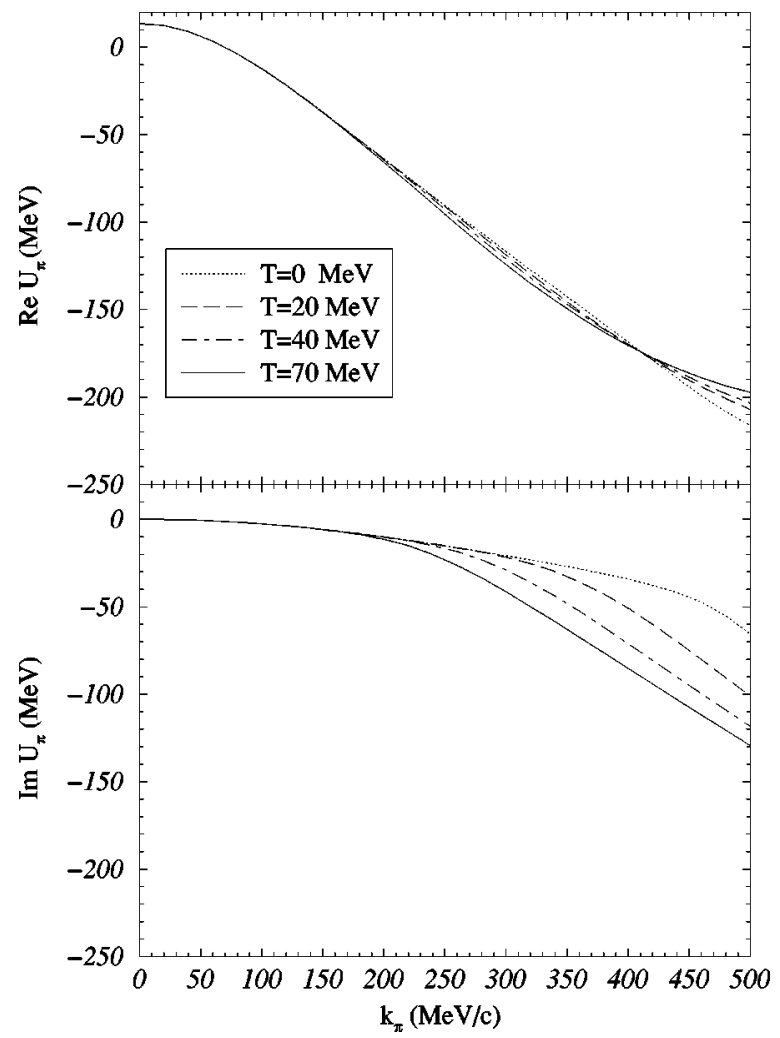

FIG. 3. Real and imaginary parts of $U_{\pi}$ at $\rho=0.17 \mathrm{fm}^{-3}$ as functions of the pion momentum for various temperatures.

the location of the peak differs more markedly. Similarly, the width (or height) of the peak for the different temperatures cannot be directly calculated from $\operatorname{Im} U_{\pi}$, but has to be obtained from $\operatorname{Im} \Pi_{\pi}$ at the quasiparticle energy.

Once the pion self-energy is introduced in the calculation of the $\bar{K}$ optical potential, its effects can be studied by comparing the results obtained by dressing only the $\bar{K}$ mesons with those in which not only the $\bar{K}$ mesons but also the pions in the intermediate meson-baryon states are dressed. In Fig. 4 , the real and imaginary parts of the $\bar{K}$ optical potential at nuclear saturation density are shown as functions of the antikaon momentum $k_{\bar{K}}$ for different temperatures. On the left panels, only the $\bar{K}$ mesons have been dressed, while the results on the right panels incorporate, in addition, the dressing of the pions. Dotted lines correspond to $T=0$ and solid lines to $T=70 \mathrm{MeV}$. At $T=0$ we find the same qualitative effects from dressing the pions as those found by the chiral model [6] shown in Ref. [29]. When the pions are dressed, the real part of the antikaon potential becomes less attractive and the imaginary part loses structure. This behavior is a direct consequence of a smoother energy dependence of the $\bar{K} N$ effective interaction when the pions are dressed (see Fig. 4 in Ref. [6]), as well as to the fact that, due to the less attractive antikaon potential, one explores this interaction at higher energies, further away from the resonant structure. When the temperature increases, the optical potential loses attraction and absorptive power, although the effect is moderate. The reason is obvious: as can be seen from Eq. (17), and assum- 


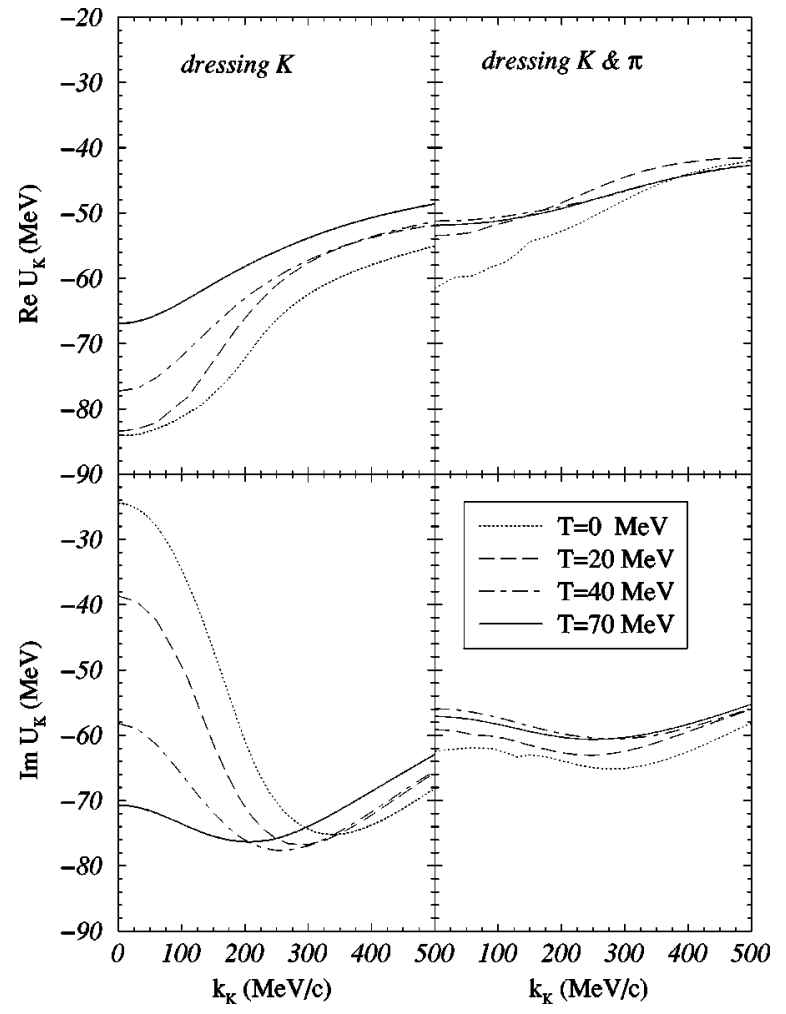

FIG. 4. Real and imaginary parts of $U_{\bar{K}}$ at $\rho=0.17 \mathrm{fm}^{-3}$ as functions of the antikaon momentum for several temperatures, dressing only $\bar{K}$ (left panels) and dressing both $\bar{K}$ and $\pi$ (right panels).

ing a weak dependence of the effective interaction $G_{\bar{K} N}$ on temperature, at a nonvanishing $T$ one is averaging over higher momentum states, where this interaction is weaker. Nevertheless, at sufficient high $T$ (see the $70-\mathrm{MeV}$ results in the right panels), one starts to gain some attraction and absorption. Evidently, this is a consequence of the $T$ dependence of the effective interaction. To visualize this additional dependence on $T$, in Fig. 5 we show the effective $\bar{K} N$ interaction for the channels $L=0, I=0$ and $L=0, I=1$, at zero center-of-mass momentum, for two different temperatures $T=0$ (dotted lines) and $T=70 \mathrm{MeV}$ (solid lines). The general trends of the effective $\bar{K} N$ interaction at these two temperatures are similar, but there are small differences that explain the behavior observed in the previous figure for the $\bar{K}$ optical potential. Indeed, the $\bar{K} N$ amplitudes involved in the construction of the $\bar{K}$ optical potential correspond to energies above $1300 \mathrm{MeV}$ and, in that region, the magnitude of the real part of the $T=70$ amplitudes is larger than the $T=0$ ones, thereby compensating for the loss of attraction induced by the higher relative momentum components present in the $\bar{K}$ optical potential at finite $T$.

It is especially interesting to observe the structure in the $I=0$ amplitude appearing below the $\pi \Sigma$ threshold, a region in energy not explored in previous works $[5,6,8]$. It appears that the resonance in the medium, previously identified with the bump in the imaginary part around $1400 \mathrm{MeV}$, might be more appropriately identified with the more pronounced peak

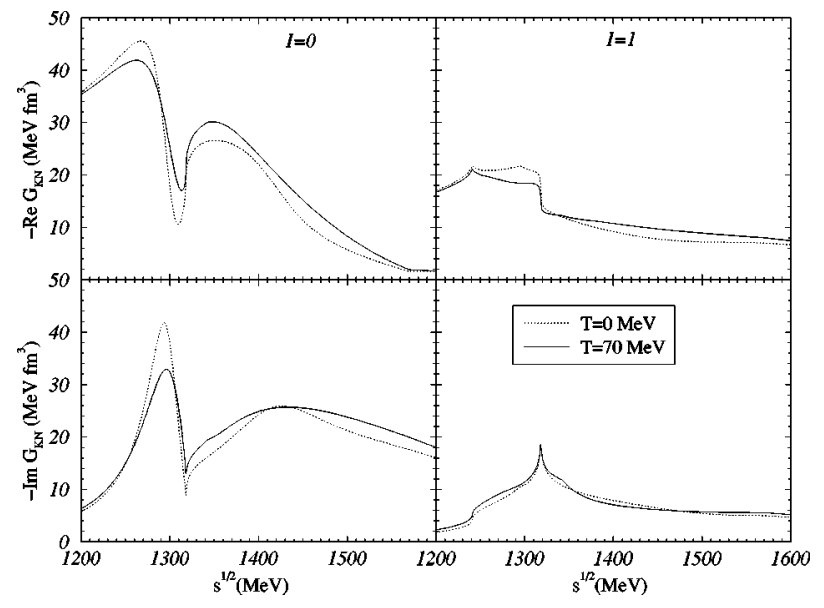

FIG. 5. Real and imaginary parts of the $\bar{K} N$ amplitude in the $I$ $=0, L=0$ channel (left panels) and the $I=1, L=0$ channel (right panels) at $\rho=0.17 \mathrm{fm}^{-3}$ as functions of the center-of-mass energy at total momentum $\left|\vec{k}_{\bar{K}}+\vec{k}_{N}\right|=0$ for $T=0$ and $70 \mathrm{MeV}$.

appearing around $1300 \mathrm{MeV}$. Whether this is a new resonance (an additional pole in the complex plane) or just a reflection of the same one but distorted by the presence of a cusp at the $\pi \Sigma$ threshold is something that deserves further study. At the moment, we can only say that there is an enhanced probability of finding a state with $\Lambda$-like quantum numbers around $1300 \mathrm{MeV}$. We note that this could be signaled by a pole in the real axis if neither the pions nor the antikaons are dressed, since in this case there would not be allowed states to decay to. In the self-consistent many-body approach used here, the states to which this peaked structure can decay are of the type $\pi(p h) \Sigma$ or $\bar{K}(\Lambda h \pi) N$, where in parentheses we have denoted an example for the component of the $\pi$ and $\bar{K}$ mesons that could show up at energies below the $\pi \Sigma$ threshold.

We have also studied the contributions of angular momentum components larger than $L=0$ to the antikaon optical potential, as we did in our $T=0$ work [8]. In Fig. 6, we display the contribution of the different partial waves to the real and imaginary parts of the $\bar{K}$ optical potential at nuclear saturation density and a temperature of $T=70 \mathrm{MeV}$. We observe that adding the higher partial waves to the $L=0$ contribution produces significant changes. The momentum dependence of the $\bar{K}$ optical potential becomes smoother, the real part becomes more attractive, and the imaginary part increases by about $25 \%$ at $k_{\bar{K}}=0$ and by $50 \%$ at momenta around $500 \mathrm{MeV} / \mathrm{c}$.

In Fig. 7 we plot the real and imaginary parts of $U_{\bar{K}}$ as functions of temperature for different densities. It is interesting to observe that $U_{\bar{K}}$ depends very weakly on temperature and stays attractive over the whole range of temperatures explored. This is qualitatively different from the results shown in Fig. 2 of Ref. [7], where the potential becomes repulsive at a finite temperature of $T=30 \mathrm{MeV}$ for $\rho=\rho_{0}$. We note that, although in that work a self-consistent scheme was also applied, the only source of medium effects in their Fig. 2 is the Pauli blocking of the nucleons in the intermediate states. As noted in Ref. [7], the transition from attraction 


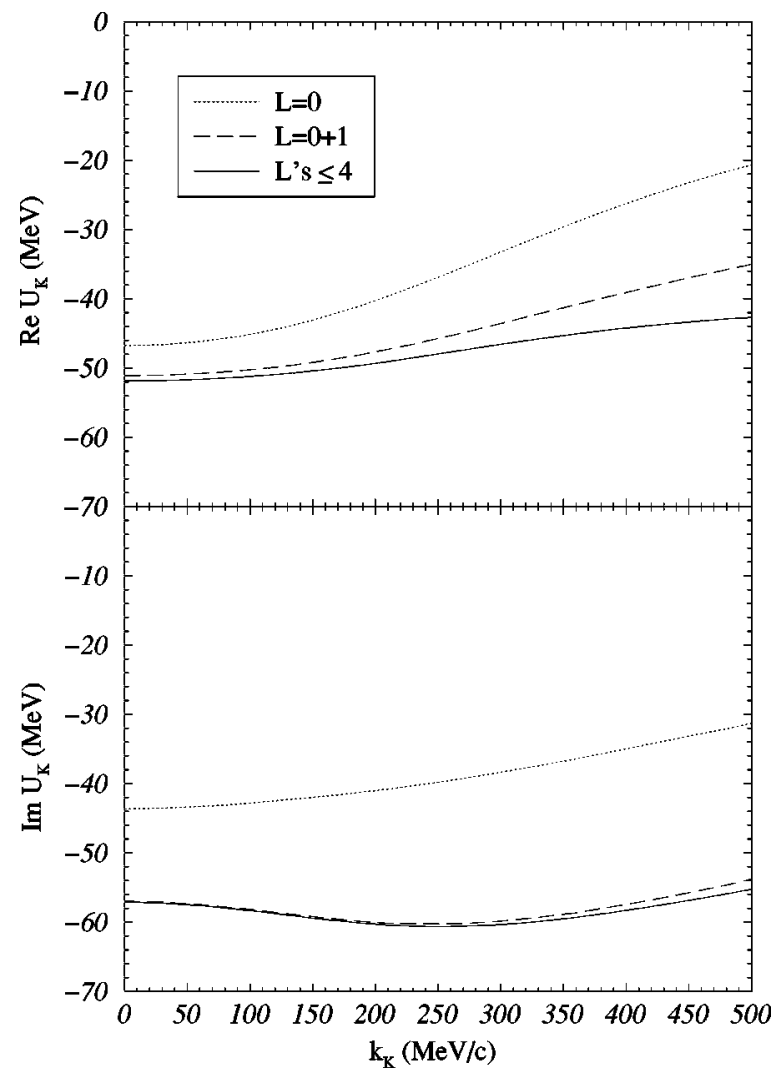

FIG. 6. Partial wave contributions to the real and imaginary parts of the $\bar{K}$ optical potential at $\rho=0.17 \mathrm{fm}^{-3}$ as functions of the antikaon momentum for $T=70 \mathrm{MeV}$.

at $T=0$ to repulsion at finite $T$ is in fact a consequence of the weakened Pauli blocking effects associated with a thermal smearing of the Fermi surface, such that eventually one is recovering the $\rho=0$ repulsive behavior. We have checked that, under the same conditions, we obtain the same qualitative behavior. Therefore, the results shown in Fig. 7 demonstrate that self-consistency effects have a tremendous influence on the behavior of the $\bar{K}$ optical potential with temperature. In particular, for temperatures as high as 70 $\mathrm{MeV}$, the real part of $U_{\bar{K}}\left(k_{\bar{K}}=0\right)$ at $\rho_{0}$ is very similar to that at $T=0$, having lost only about $10 \mathrm{MeV}$ of attraction. Hence, our results, based on a fully self-consistent calculation, do not support the claims that the $\bar{K}$ optical potential might be repulsive at finite $T$. The attraction found here for the $\bar{K}$ optical potential at finite $\rho$ and $T$, together with enhanced in-medium $K^{-}$production cross sections $[7,19]$, may help to explain the enhanced $K^{-} / K^{+}$ratio measured at GSI by the KaoS Collaboration [14].

Finally, in Fig. 8 we present the $\bar{K}$ spectral function as a function of energy for two momentum values $k_{\bar{K}}=0$ and 400 $\mathrm{MeV} / c$, at $T=0$ (dotted lines) and $T=70 \mathrm{MeV}$ (solid lines). The structures observed on the left-hand side of the peaks, especially visible for the $T=0$ spectral functions, are due to the excitation of hyperon-hole states with $\bar{K}$ quantum numbers that are present when the $L=1$ components of the $\bar{K} N$ interaction are incorporated. We note, however, that the high-

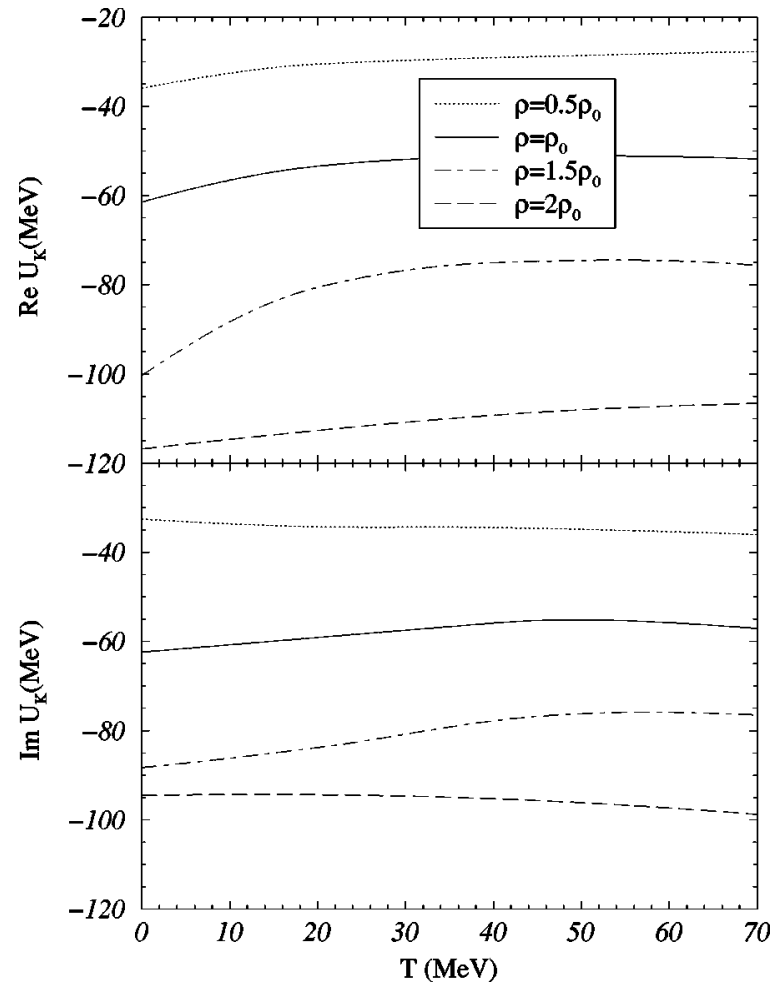

FIG. 7. Real and imaginary parts of the $\bar{K}$ optical potential as functions of the temperature for different densities.

est peak observed in the $T=0$ spectral function for $k_{\bar{K}}=0$ corresponds to the enhancement observed in the $I=0 \bar{K} N$ amplitude below the $\pi \Sigma$ threshold. The inclusion of a finite $T$ washes most of these structures out, and the resulting spectral functions show basically a single pronounced peak.

\section{CONCLUSIONS}

We have studied the $\bar{K} N$ interaction in hot and dense matter by extending to finite $T$ our previous $T=0$ model, which

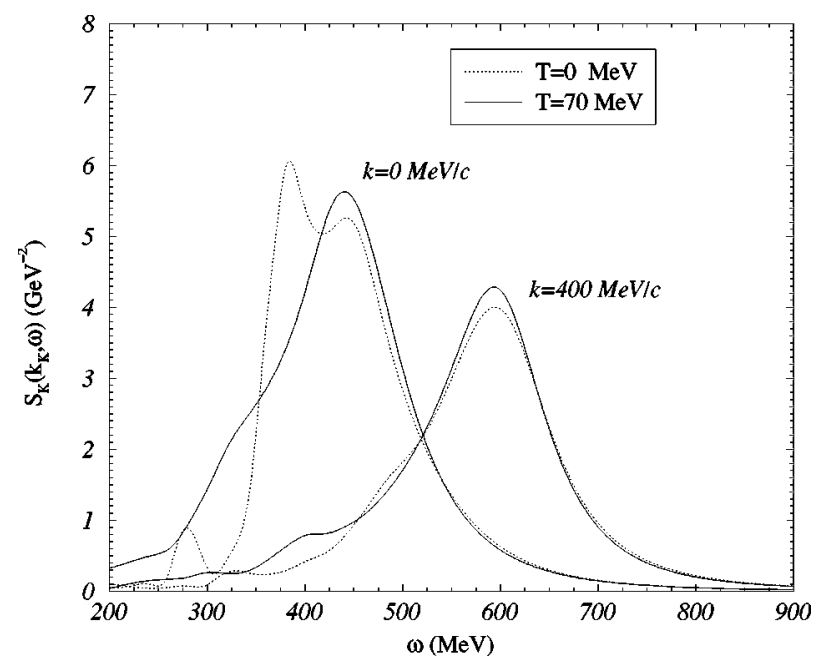

FIG. 8. Spectral density of the antikaon at $\rho=0.17 \mathrm{fm}^{-3}$ as a function of energy for antikaon momenta $k_{\bar{K}}=0$ and $400 \mathrm{MeV} / c$, and for $T=0$ and $70 \mathrm{MeV}$. 
is based on a self-consistent coupled-channel calculation taking, as bare interaction, the meson-exchange potential of the Jülich group. Although more moderate than in the $T=0$ case, we have also found at finite temperature that dressing the pions in the intermediate $\pi Y$ loops has a strong influence on the $\bar{K} N$ amplitudes and, consequently, on the $\bar{K}$ optical potential.

Partial waves higher than the $L=0$ component of the $\bar{K} N$ effective interaction contribute significantly to the antikaon optical potential at finite temperature. The real part gains attraction and the imaginary part becomes more absorptive. At a momentum of $500 \mathrm{MeV} / c$, the contribution of the $L$ $>0$ components is as large as that of the $L=0$ ones.

We have found that self-consistency effects have a tremendous influence on the behavior of the antikaon optical potential with temperature. At a normal saturation density, the optical potential remains attractive for temperatures as large as $70 \mathrm{MeV}$, contrary to what would be observed if only Pauli blocking medium effects were considered, which would give a repulsive optical potential at these high temperatures.

In general, temperature effects smear out the different observables with respect to the $T=0$ case. For instance, the antikaon spectral function at finite temperature shows much less structure than that at $T=0$, reducing its shape basically to a peak located at the quasiparticle energy.

The attractive potential found here for finite density, finite temperature, and finite momentum, i.e. for the typical scenario found in heavy-ion collisions at GSI, is especially interesting to understand the enhanced $K^{-} / K^{+}$ratio measured by the KaoS Collaboration at GSI, together with other mechanisms that have been already suggested in the literature, such as an enhanced production of $K^{-}$through $\pi Y$ collisions. Work along these lines is in progress.

\section{ACKNOWLEDGMENTS}

We are very grateful to Dr. Jürgen Schaffner-Bielich and Dr. Wolfgang Cassing for useful discussions. L.T. wishes to acknowledge the hospitality extended to her at Brookhaven National Laboratory. This work was partially supported by DGICYT Project No. PB98-1247 and by the Generalitat de Catalunya Project No. 2000SGR00024. L.T. also wishes to acknowledge support from the Ministerio de Educación y Cultura (Spain).

\section{APPENDIX}

In this appendix we show how to obtain the pion selfenergy at finite temperature. First we will review the formalism at $T=0$.

When the pion propagates in nuclear matter, it scatters with the surrounding nucleons and the basic first order response of the medium to the propagation of the pion field is the excitation of a particle-hole pair. In the region of intermediate energies $\left(T_{\pi}=0-300 \mathrm{MeV}\right), \Delta-h$ excitation also takes place due to the excitation of internal degrees of freedom of the nucleon. Therefore, to obtain the pion self-energy in symmetric nuclear matter at $T=0$, both effects have to be taken into account. We follow the nomenclature, parameters and approximations mentioned in the Appendix of Ref. [30]. According to this reference, the two contributions to the pion self-energy can be written as

$$
\begin{gathered}
\Pi\left(\vec{q}, q^{0}\right)=\left(\frac{f_{N}}{m_{\pi}}\right)^{2} \vec{q}^{2} U\left(\vec{q}, q^{0}\right), \\
U\left(\vec{q}, q^{0}\right)=U_{N}\left(\vec{q}, q^{0}\right)+U_{\Delta}\left(\vec{q}, q^{0}\right),
\end{gathered}
$$

where $U\left(\vec{q}, q^{0}\right)$ is the sum of $1 p-1 h$ and $1 \Delta-1 h$ Lindhard functions, which at $T=0$ read

$$
\begin{aligned}
U_{N}\left(\vec{q}, q^{0}\right)= & \nu_{N} \int \frac{d^{3} k}{(2 \pi)^{3}}\left[\frac{\theta\left(k_{F}-|\vec{k}|\right)\left[1-\theta\left(k_{F}-|\vec{k}+\vec{q}|\right)\right]}{q^{0}+E_{N}(\vec{k})-E_{N}(\vec{k}+\vec{q})+i \eta}\right. \\
& \left.+\frac{\theta\left(k_{F}-|\vec{k}+\vec{q}|\right)\left[1-\theta\left(k_{F}-|\vec{k}|\right)\right]}{-q^{0}-E_{N}(\vec{k})+E_{N}(\vec{k}+\vec{q})+i \eta}\right],
\end{aligned}
$$

$$
\begin{aligned}
U_{\Delta}\left(\vec{q}, q^{0}\right) & \\
= & \nu_{\Delta} \int \frac{d^{3} k}{(2 \pi)^{3}}\left[\frac{\theta\left(k_{F}-|\vec{k}|\right)}{q^{0}+E_{N}(\vec{k})-E_{\Delta}(\vec{k}+\vec{q})+i \frac{\Gamma\left(\vec{q}, q^{0}\right)}{2}}\right. \\
& \left.+\frac{\theta\left(k_{F}-|\vec{k}+\vec{q}|\right)}{-q^{0}+E_{N}(\vec{k}+\vec{q})-E_{\Delta}(\vec{k})+i \frac{\Gamma\left(\vec{q},-q^{0}\right)}{2}}\right],
\end{aligned}
$$

where $\nu_{N}$ and $\nu_{\Delta}$ contain the sum over spin-isospin degrees of freedom in symmetric nuclear matter. In the case of $1 \Delta$ $-1 h$, it also includes the decay constant $f_{\Delta}^{*}$ fitted experimentally. Hence

$$
\begin{gathered}
\nu_{N}=4 \\
\nu_{\Delta}=\frac{16}{9}\left(\frac{f_{\Delta}^{*}}{f_{N}}\right)^{2}, \quad \frac{f_{\Delta}^{*}}{f_{N}}=2.13 .
\end{gathered}
$$

The quantity $\Gamma\left(\vec{q}, \pm q^{0}\right)$ is the $\Delta$ width, which we take to depend only on the external variables of the pion, as in Ref. [30].

The definitions of the Lindhard functions [Eqs. (A2) and (A3)] have been written explicitly here in order to facilitate the comparison with the finite $T$ case. On the other hand, the analytical results can be found in the Appendix of Ref. [30]. We also include in the pion self-energy the coupling to $2 p$ $-2 h$ excitations, following the phase-space approach of Ref. [31].

Once $1 p-1 h(2 p-2 h)$ and $1 \Delta-1 h$ excitations are computed, form factors and short-range correlations are introduced. One pion exchange provides a good description of the nucleon-nucleon interaction for long distances, but this is 
not the only ingredient. At shorter distances other effects must be considered, such as correlated and uncorrelated two pion exchange and the exchange of heavier mesons. The final expression for the pion self-energy reads

$$
\begin{gathered}
\Pi\left(\vec{q}, q^{0}\right)=F\left(\vec{q}, q^{0}\right)\left(\frac{f_{N}}{m_{\pi}}\right)^{2} \vec{q}^{2} \frac{U\left(\vec{q}, q^{0}\right)}{1-\left(\frac{f_{N}}{m_{\pi}}\right)^{2} g^{\prime} U\left(\vec{q}, q^{0}\right)}, \\
U\left(\vec{q}, q^{0}\right)=U_{N}\left(\vec{q}, q^{0}\right)+U_{\Delta}\left(\vec{q}, q^{0}\right),
\end{gathered}
$$

where $F\left(\vec{q}, q^{0}\right)$ is the form factor,

$$
\begin{aligned}
F\left(\vec{q}, q^{0}\right) & =\left(\frac{\Lambda^{2}-m_{\pi}^{2}}{\Lambda^{2}-\left(q^{02}-\vec{q}^{2}\right)}\right)^{2}, \\
\Lambda & =1200 \mathrm{MeV},
\end{aligned}
$$

and $g^{\prime}$ the Landau-Migdal parameter taken from the particlehole interaction described in Ref. [32], which includes $\pi$ and $\rho$ exchange modulated by the effect of nuclear short-range correlations.

The pion self-energy also includes an $s$-wave piece

$$
\Pi(\rho)_{s \text {-wave }}=-4 \pi\left(1+\frac{m_{\pi}}{m_{N}}\right) b_{0} \rho,
$$

with $b_{0}=-0.0285 / m_{\pi}$, taken from the parametrization of Ref. [33], which is equivalent to the results of Ref. [34].

\section{$1 p-1 h$ and $1 \Delta-1 h$ Lindhard functions at finite temperature}

The effect of temperature in the pion self-energy comes from the modification of the Fermi sea. At a given temperature, nucleons are distributed following the corresponding Fermi-distribution. Then, $U\left(\vec{q}, q^{0}\right)$ transforms into

$$
U\left(\vec{q}, q^{0}, T\right)=U_{N}\left(\vec{q}, q^{0}, T\right)+U_{\Delta}\left(\vec{q}, q^{0}, T\right),
$$

with

$$
\begin{aligned}
U_{N}\left(\vec{q}, q^{0}, T\right)= & \nu_{N} \int \frac{d^{3} k}{(2 \pi)^{3}}\left[\frac{n(\vec{k}, T)[1-n(\vec{k}+\vec{q}, T)]}{q^{0}+E_{N}(\vec{k})-E_{N}(\vec{k}+\vec{q})+i \eta}\right. \\
& \left.+\frac{n(\vec{k}+\vec{q}, T)[1-n(\vec{k}, T)]}{-q^{0}-E_{N}(\vec{k})+E_{N}(\vec{k}+\vec{q})+i \eta}\right]
\end{aligned}
$$

and

$$
\begin{aligned}
& U_{\Delta}\left(\vec{q}, q^{0}, T\right) \\
& =\nu_{\Delta} \int \frac{d^{3} k}{(2 \pi)^{3}}\left[\frac{n(\vec{k}, T)}{q^{0}+E_{N}(\vec{k})-E_{\Delta}(\vec{k}+\vec{q})+i \frac{\Gamma\left(q^{0}, \vec{q}\right)}{2}}\right. \\
& \quad+\frac{n(\vec{k}+\vec{q}, T)}{\left.-q^{0}+E_{N}(\vec{k}+\vec{q})-E_{\Delta}(\vec{k})+i \frac{\Gamma\left(\vec{q},-q^{0}\right)}{2}\right],}
\end{aligned}
$$

where the step function $\theta$ for nucleons has been substituted by the distribution $n(\vec{k}, T)$ at the corresponding temperature.

The imaginary part of $U_{N}\left(\vec{q}, q^{0}, T\right)$ at finite temperature can be obtained analytically,

$$
\begin{aligned}
& \operatorname{Im} U_{N}\left(\vec{q}, q^{0}, T\right)=\operatorname{Im} U_{N}^{D}\left(\vec{q}, q^{0}, T\right)+\operatorname{Im} U_{N}^{C}\left(\vec{q}, q^{0}, T\right) \\
& =\operatorname{Im} U_{N}^{D}\left(\vec{q}, q^{0}, T\right)\left(1+e^{-q^{0} / T}\right), \\
& \left.\operatorname{Im} U_{N}\left(\vec{q}, q^{0}, T\right)=-\frac{3}{2} \pi \rho \frac{m_{\pi}^{2} T}{k_{F}^{3}|\vec{q}|} \ln \frac{1-n\left(p^{+}, T\right)}{1-n\left(p^{-}, T\right)} \operatorname{cotanh} \mid \frac{q^{0}}{2 T}\right), \\
& \qquad p^{+}=\frac{m 11)}{|\vec{q}|}\left|q^{0}+\frac{\vec{q}^{2}}{2 m}\right|, \quad p^{-}=\frac{m_{\pi} \mid}{|\vec{q}|}\left|q^{0}-\frac{\vec{q}^{2}}{2 m_{\pi}}\right|, \quad \text { (A12) } \\
& \text { where Im } U_{N}^{D}\left(\vec{q}, q^{0}, T\right) \text { and Im } U_{N}^{C}\left(\vec{q}, q^{0}, T\right) \text { are the imaginary } \\
& \text { parts of the direct and crossed contributions }[\text { first and second } \\
& \text { terms on the right-hand side of Eq. }(\text { A9), respectively]. } \\
& \text { The real part of } U_{N}\left(\vec{q}, q^{0}, T\right) \text { is obtained via the dispersion } \\
& \text { relation } \\
& \qquad-\frac{1}{\pi} P \int d \omega^{\prime} \frac{\operatorname{Im} U_{N}^{C}\left(\omega^{\prime}, \vec{q}, T\right)}{-q^{0}+\omega^{\prime}}, \\
& \text { Re } U_{N}\left(\vec{q}, q^{0}, T\right)=-\frac{1}{\pi} P \int d \omega^{\prime} \frac{\operatorname{Im} U_{N}^{D}\left(\omega^{\prime}, \vec{q}, T\right)}{q^{0}-\omega^{\prime}}
\end{aligned}
$$

or, in a more compact form,

$$
\operatorname{Re} U_{N}\left(\vec{q}, q^{0}, T\right)=-\frac{1}{\pi} P \int d \omega^{\prime} \frac{\operatorname{Im} U_{N}\left(\omega^{\prime}, \vec{q}, T\right) \tanh \left(\frac{\omega^{\prime}}{2 T}\right)}{q^{0}-\omega^{\prime}}
$$

Equations (A12) and (A14) define the nucleon Lindhard function at finite $T$. 
As mentioned in the appendix of Ref. [30], the $1 \Delta-1 \mathrm{~h}$ Lindhard function at $T=0, U_{\Delta}\left(\vec{q}, q^{0}\right)$, can be derived analytically by neglecting the difference of $\vec{k}^{2}$ terms in

$E_{N}(\vec{k})-E_{\Delta}(\vec{k} \pm \vec{q})=\frac{\vec{k}^{2}}{2 m_{N}}-\frac{\vec{k}^{2}}{2 m_{\Delta}}-\frac{\vec{q}^{2}}{2 m_{\Delta}} \mp \frac{\vec{k} \vec{q}}{m_{\Delta}}+m_{N}-m_{\Delta}$,

and assuming that the $\Delta$ width only depends on the external variables. We perform the same approximations at finite $T$. Redefining $\vec{k}+\vec{q} \rightarrow-\vec{k}$ in the second term on the right-hand side of Eq. (A10), such that it becomes the same as the first one but changing $q^{0} \rightarrow-q^{0}$, we finally arrive at

$$
\begin{aligned}
U_{\Delta}\left(\vec{q}, q^{0}, T\right)= & \frac{2}{3}\left(\frac{f_{\Delta}^{*}}{f_{N}}\right)^{2} \frac{m_{\Delta} \rho}{q k_{F}^{3}} \int d k k n(k, T)\left[\ln \left(\frac{z^{+}+1}{z^{+}-1}\right)\right. \\
& \left.+\ln \left(\frac{z^{-}+1}{z^{-}-1}\right)\right] \\
z^{ \pm}= & \frac{m_{\Delta}}{q k}\left( \pm q^{0}-\frac{\vec{q}^{2}}{2 m_{\Delta}}-\left(m_{\Delta}-m_{N}\right)+i \frac{\Gamma\left( \pm q^{0}, \vec{q}\right)}{2}\right) .
\end{aligned}
$$

[1] See state-of-the-art experimental information on the web sites: www.gsi.de, www.cern.ch, www.bnl.gov/rhic

[2] D. B. Kaplan and A. E. Nelson, Phys. Lett. B 175, 57 (1986); 179, 409(E) (1986).

[3] A. Ramos, J. Schaffner-Bielich, and J. Wambach, in Physics of Neutron Star Interiors, edited by D. Blaschke, N. K. Glendenning, and A. Sedrakian, Lecture Notes in Physics Vol. 578 (Springer, New York, 2001), p. 175.

[4] E. Friedman, A. Gal, and C. J. Batty, Nucl. Phys. A579, 518 (1994).

[5] M. Lutz, Phys. Lett. B 426, 12 (1998).

[6] A. Ramos and E. Oset, Nucl. Phys. A671, 481 (2000).

[7] J. Schaffner-Bielich, V. Koch, and M. Effenberg, Nucl. Phys. A669, 153 (2000).

[8] L. Tolós, A. Ramos, A. Polls, and T. T. S. Kuo, Nucl. Phys. A690, 547 (2001).

[9] E. Oset and A. Ramos, Nucl. Phys. A635, 99 (1998).

[10] S. Hirenzaki, Y. Okumura, H. Toki, E. Oset, and A. Ramos, Phys. Rev. C 61, 055205 (2000).

[11] A. Baca, C. García-Recio, and J. Nieves, Nucl. Phys. A673, 335 (2000).

[12] A. Cieplý, E. Friedman, A. Gal, and J. Mares, Nucl. Phys. A696, 173 (2001).

[13] N. Kaiser, P. B. Siegel, and W. Weise, Nucl. Phys. A594, 325 (1995); N. Kaiser, T. Waas, and W. Weise, ibid. A612, 297 (1997).

[14] R. Barth et al., Phys. Rev. Lett. 78, 4007 (1997); F. Laue et al., ibid. 82, 1640 (1999).

[15] W. Cassing, E. L. Bratkovskaya, U. Mosel, S. Teis, and A.
Sibirtsev, Nucl. Phys. A614, 415 (1997); E. L. Bratkovskaya, W. Cassing, and U. Mosel, ibid. A622, 593 (1997).

[16] G. Q. Li, C.-H. Lee, and G. Brown, Nucl. Phys. A625, 372 (1997); Phys. Rev. Lett. 79, 5214 (1997).

[17] G. Q. Li and G. Brown, Phys. Rev. C 58, 1698 (1998).

[18] A. Sibirtsev and W. Cassing, Nucl. Phys. A641, 476 (1998).

[19] A. Ohnishi, Y. Nara, and V. Koch, Phys. Rev. C 56, 2767 (1997).

[20] H. Tamura et al., Phys. Rev. C 40, R479 (1989).

[21] K. Kubota et al., Nucl. Phys. A602, 327 (1996).

[22] A. Müller-Groeling, K. Holinde, and J. Speth, Nucl. Phys. A513, 557 (1990).

[23] J. Caro-Ramon, N. Kaiser, S. Wetzel, and W. Weise, Nucl. Phys. A672, 249 (2000).

[24] M. Lutz and E. Kolomeitsev, Nucl. Phys. A700, 193 (2002).

[25] M. F. M. Lutz and C. L. Korpa, Nucl. Phys. A700, 309 (2002).

[26] S. Balberg and A. Gal, Nucl. Phys. A625, 435 (1997).

[27] R. Machleidt, Adv. Nucl. Phys. 19, 189 (1989).

[28] B. D. Serot and J. D. Walecka, Adv. Nucl. Phys. 16, 12 (1986).

[29] A. Ramos, S. Hirenzaki, S. S. Kamalov, T. T. S. Kuo, Y. Okumura, E. Oset, A. Polls, H. Toki, and L. Tolós, Nucl. Phys. A691, 258c (2001).

[30] E. Oset, P. Fernández de Córdoba, L. L. Salcedo, and R. Brockmann, Phys. Rep. 188, 79 (1990).

[31] A. Ramos, E. Oset, and L. L. Salcedo, Phys. Rev. C 50, 2314 (1994).

[32] E. Oset, H. Toki, and W. Weise, Phys. Rep. 83, 28 (1982).

[33] R. Seki and K. Masutani, Phys. Rev. C 27, 2799 (1983).

[34] O. Meirav, E. Friedman, R. R. Johnson, R. Olszewski, and P. Weber, Phys. Rev. C 40, 843 (1989). 